\title{
Rectal tuberculosis: the great mimic
}

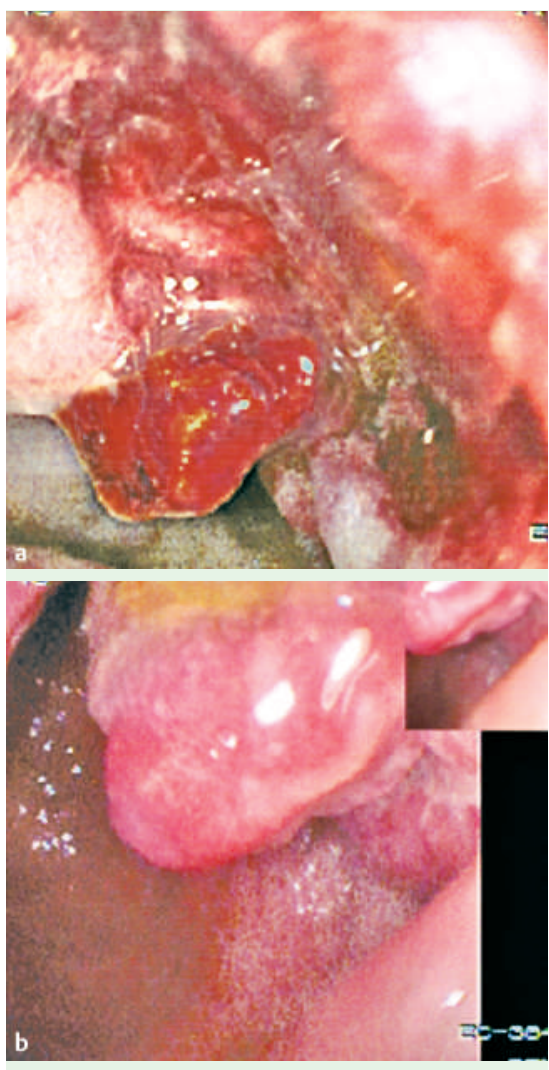

Figure $\mathbf{1}$ a, b Rectal mass.

Gastrointestinal tuberculosis (TB) is a diagnostic challenge in the absence of pulmonary infection. Gastrointestinal TB can involve any part of the alimentary tract from the mouth to the anus, the distal ileum being the most common site [1]. Lesions above and below this site are rare. A 72-year-old gentleman complaining of constipation, abdominal pain, and a 2month history of loss of appetite and weight loss was referred to our gastroenterology department.

Examination revealed pallor; vital signs including pulse, blood pressure, and respiratory rate were within normal limits. Abdominal examination demonstrated tenderness in the left iliac fossa, but no mass could be palpated. Digital rectal examination revealed a hard, palpable, fixed mass around $7 \mathrm{~cm}$ from the anal verge.

Examination of other systems gave unremarkable findings. On the basis of the history and clinical findings a provisional diagnosis of rectal carcinoma was made. Abdominal ultrasonography and chest radiography did not show any abnormali-

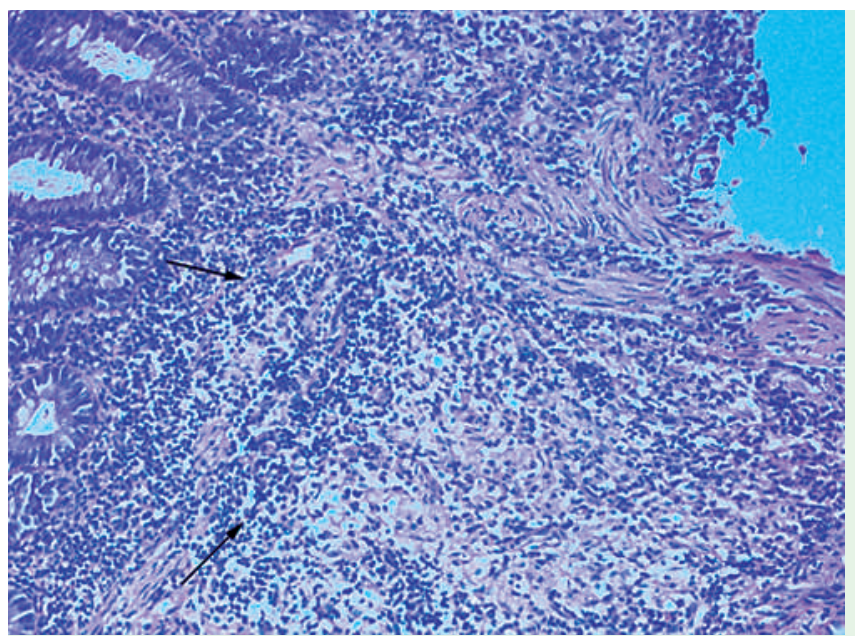

Figure 2 Granuloma with central necrosis.

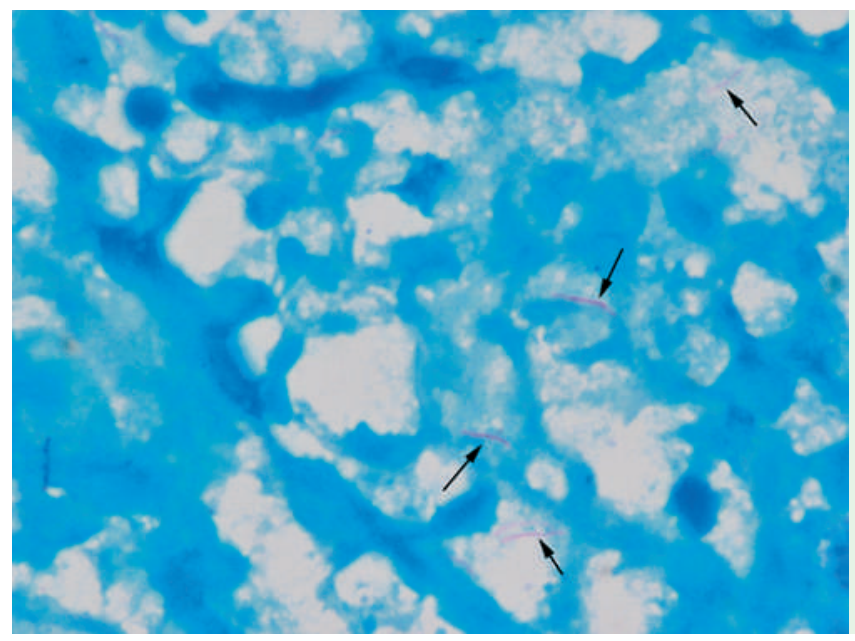

Figure 3 Multiple acid-fast bacilli.

ty. Colonoscopy showed an irregular, ulcerated, nodular mass in the rectum around $1.5 \mathrm{~cm}$ in size ( $\bullet$ Figure 1 ) while the rest of the colon appeared normal. Histopathological investigation revealed chronic inflammatory cells in the mucosa showing a number of scattered epithelioid histiocytic granulomas with central necrosis ( Figure 2), and Ziehl-Neelsen staining showed numerous acid-fast bacilli ( Figure 3). On the basis of these findings rectal tuberculosis was diagnosed, and the patient was started on an antituberculosis treatment regime with regular follow-up.

Intestinal TB commonly occurs in the second and third decades of life; only $2 \%$ of cases present after the age of 60 years [2].Only half of the patients have an abnormality in the chest radiograph or have active pulmonary tuberculosis [3]. Between $30 \%$ and $50 \%$ of intestinal cases reported did not reveal acid-fast bacilli on Ziehl-Neelsen staining [4].

This case shows that tubercular involvement of the rectum is rare. It can closely mimic carcinoma of the rectum, and its diagnosis can be challenging.

Endoscopy_UCTN_Code_CCL_1AD_2AD

\section{Kamani ${ }^{1}$, A. Ahmed ${ }^{1}$, M. Shah ${ }^{1}$, \\ S. Hasan' ${ }^{2}$ W. Jafri ${ }^{1}$}

Gastroenterology Section, Department of Medicine, Aga Khan University Hospital, Karachi, Pakistan

2 Department of Pathology, Aga Khan University Hospital, Karachi, Pakistan 
References

1 Bhansali SK. Abdominal tuberculosis. Experience with 300 cases. Am J Gastroenterol 1977; 67: 324-337

2 Sane SY, Numbkhar SA. Carcinoma of colon with tuberculosis. J Postgrad Med 1980; 26: $199-200$

3 Lingenfelser T, Zak J, Marks IN et al. Abdominal tuberculosis still a potentially lethal disease. Am J Gastroenterol 1993; 88: $744-$ 750

4 Bhargava DK, Tando HD, Chwla TC et al. Diagnosis of ileocecal and colonic tuberculosis by colonoscopy. Gastrointest Endosc 1985; 31 : $68-70$

\section{Bibliography}

DOI $10.1055 / \mathrm{s}-2007-966367$

Endoscopy 2007; 39: $227-228$

(c) Georg Thieme Verlag KG Stuttgart · New York . ISSN 0013-726X
Corresponding author

\section{Kamani FCPS, MRCP(UK)}

Gastroenterology Section

Department of Medicine Aga Khan University Hospital Pakistan

Fax: +39-49-343769

lubna.kamani@aku.edu 